\title{
Could novel diagnostic technology Cognetivity accelerate dementia research? An interview with Dr Sina Habibi
}

\author{
Sina Habibi ${ }^{1}$ \& Rachel Jenkins ${ }^{1}$
}

Accepted for publication: 14 March 2019; Published online: 24 May 2019

Keywords: clinical • dementia • early diagnosis • technology

Bio: Dr Sina Habibi is CEO and co-founder at Cognetivity Neurosciences. With a PhD in Engineering from Cambridge University, Sina has in-depth experience in research involving nano-technology and synthetic biology, product management and technology consultancy.

\section{Questions}

Please introduce yourself to our readers \& tell them about your career to date, including what your current role as Cognetivity CEO involves

I am a mechanical engineer with an $\mathrm{MSc}$ and $\mathrm{PhD}$ from the University of Cambridge. I have worked previously as a senior researcher in nano-biotechnology and in various areas of technology consultancy. The essence of my educational and professional life is analytical problem-solving. Through my diverse experience in various fields such as nanofabrication, advanced microscopy and entrepreneurship, I have become proficient in numerical and statistical data collection and analysis which I am applying to the field of dementia.

Founded in 2013, Cognetivity Neurosciences is based on original research carried out at the University of Cambridge by my co-founder, Seyed Khaligh-Razavi. Dr Khaligh-Razavi is a computational neuroscientist and his research was originally looking into how the human brain is able to process extremely complex visual information in a very short period of time, and how to measure this effectively. From this research came the idea that this approach would be a highly effective way of measuring changes in cognitive performance associated with disease, and the original design of Cognetivity's ICA test was developed from these principles.

As CEO, I aim to provide the vision for the company. I help to define our objectives to achieve this vision while promoting our company's culture. Through recruiting the right people and inspiring my colleagues, I ensure we are all working toward the same goal.

DPUK has recently announced its new partnership with Cognetivity. Please could you explain to our readers what Cognetivity does \& why DPUK has partnered with them?

Cognetivity Neurosciences provides early (preclinical) detection and remote monitoring of dementia using artificial intelligence and the latest neuroscience. DPUK is a platform whereby patients, pharma, medical device companies and research institutes all come together to share knowledge, expertise and research data to understand and tackle dementia. Our unique solution helps researchers to measure cognitive performance objectively, repeatedly and without typical education, culture and learning bias, all of which have previously resulted in considerable limitations in dementia research.

How do you think platforms like the 'Integrated Cognitive Assessment' offered by Cognetivity will help the fight against dementia?

We have developed an image-based cognitive function test that avoids the confounding biases inherent to standard tests and uses artificial intelligence (AI) to interpret the results. The 'Integrated Cognitive Assessment' (ICA), described in Scientific Reports, could enable better detection of cognitive impairment in Alzheimer's disease and other neurodegenerative conditions, leading to much earlier diagnosis for patients.

Future Medicine 
Early diagnosis can seem to be a contentious topic, but there are some key benefits provided by such a diagnosis which can greatly help in the fight against dementia.

First, those that have received an early diagnosis can make informed lifestyle changes to delay the onset of later stage symptoms. A delay of even 5 years in the onset of symptoms will reduce morbidity rates significantly and increase our patients' quality of life. With the associated specialist treatment given to early diagnosis patients, mortality rates can also be reduced when the patient reaches the advanced stages of the condition.

A major benefit that our test provides in the fight against dementia is that it allows research to be conducted at the earliest stages of the condition, both furthering our understanding and allowing for new treatments to be tested and tracked. While there is currently no cure, it will be crucial to administer such treatment as early as possible before permanent cognitive impairment occurs, making an early diagnosis tool an important part in tackling this condition.

Finally, a number of studies have shown that through delayed admissions to healthcare services, massive economic savings could be made. The total number of hospital admissions would be reduced, meaning reduced pressure on healthcare services and large savings in the public and private sectors.

\section{What do you consider to be the greatest challenges hindering dementia research \& do you think we will be able to overcome them?}

To date, there are four drugs approved and in routine use for the symptomatic treatment of AD (years denote US FDA approval date): Donepezil (1997), Rivastigmine (2000), Galantamine (2001) and Memantine (2003).

There are two important points here. First, while beneficial, modest improvements in cognition, global cognitive status and functional ability have been demonstrated), all of the above provide only partial symptomatic relief for patients and while disease modification is too short to be genuinely considered as truly effective. These treatments aim to ameliorate cognitive function via modulation of remaining circuitry, either via agonism of the cholinergic system, or the antagonism of N-methyl-D-aspartate receptors. However, neurodegeneration continues unabated despite these therapies.

Second, in the period of 2004-2019, not a single novel compound (whether symptomatic or disease modifying) has gained regulatory approval. Therefore, in spite of the 635 interventional clinical trials (139 Phase III, 266 Phase II and 267 Phase I) carried out between 2004 and 2019, and high levels of investment (a recent estimate suggests that with existing infrastructures an $\mathrm{AD}$ drug discovery program costs approximately $\$ 5.69$ billion from start to end), the failure rate over the preceding 15 years sits at $100 \%$. Clearly, with a lack of discernible success, our current approaches and infrastructures require improvement and rethinking. The underlying causes remain contentious, and many issues remain unaddressed. We believe particular attention should be paid to clinical trial recruitment via more sophisticated and cost-effective patient characterization and clinical trial end points.

Another major challenge is that diagnostic scores from standard cognitive function tests, including the usually paper-based Montreal Cognitive Assessment (MoCA) and Addenbrooke's Cognitive Examination-Revised MiniACE (M-ACE) tests, can be skewed by the test taker's language and education level, with high education levels correlating with better scores.

To address these issues, the Cognetivity team created the ICA, a digital test that is administered on an iPad, web or Raspberry Pi-based platform. ICA has subjects classify pictures, displayed in random order, as containing or lacking animals to generate a score based on response times and accuracies. Next, an AI engine analyzes the subject's score, reaction times and responses, and demographic information, including age and sex, to predict cognitive status.

\section{Leading on from the challenges, where do you see the field of dementia research in the next $5-10$ years?}

Recently, we have observed an increased diversification in research and treatment strategies, moving away from perhaps an excessive focus on amyloid in the preceding 20 years, and I expect this trend to continue over the next 5-10 years. Hopefully, a continuation in this trend will lead to a better understanding of the heterogeneity of the disease, the discovery of novel drug targets and lead to promising therapeutics entering the clinic.

Translating this research into successful clinical trials and ultimately approved drugs may, however, remain a significant obstacle. Without improvements in our ability to diagnose patients early, to effectively identify patients for clinical trial recruitment and without reliable clinical trial end points, these trials may still result in failure. We 
are, however, optimistic that the implementation of digital solutions such as our own ICA, likely coupled with an improved understanding of biomarkers, may go some way to addressing these issues.

\section{Disclaimer}

The opinions expressed in this interview are those of the interviewee and do not necessarily reflect the views of Future Medicine Ltd.

\section{Financial \& competing interests disclosure}

$\mathrm{S}$ Habibi is a director and shareholder in Cognetivity. The author has no other relevant affiliations or financial involvement with any organization or entity with a financial interest in or financial conflict with the subject matter or materials discussed in the manuscript. This includes employment, consultancies, honoraria, stock ownership or options, expert testimony, grants or patents received or pending, or royalties.

No writing assistance was utilized in the production of this manuscript.

\section{Open access}

This work is licensed under the Creative Commons Attribution-NonCommercial-NoDerivatives 4.0 Unported License. To view a copy of this license, visit http://creativecommons.org/licenses/by-nc-nd/4.0/. 
